\section{Brain Tumor Imaging}

R. Jain, M. Essig, eds.

New York, New York: Thieme, 2016, 254 pages, \$149.99

As stated in the preface, Brain Tumor Imaging is a broad overview of the currently available diagnostic methods for the complex management of brain tumors, allowing the reader to learn about their imaging features and the most significant differentials.

The book consists of 19 chapters from 36 contributing authors comprising 5 professors, 7 associate professors, 8 assistant professors, 1 instructor, and 15 residents, fellows, or other types of staff physician. Chapters 1-6, 8-10, and 13-19 are on various current and future MRI techniques, mostly for applications in human gliomas, especially glioblastoma multiforme. Chapter 7 is on diffusion imaging using CT; chapter 11 is on metabolic tumor imaging using MR spectroscopy; and chapter 12 is on PET, SPECT, and hybrid imaging, including PET/MRI. The Macdonald and RANO (Revised Assessment in Neurooncology) criteria for assessing response to treatment are compared in chapter 2 . From the earlier to the later chapters, the progression of MRI is followed from its earlier use in conventional modalities to its later use in more experimental technologies (such as ultrasmall superparamagnetic iron oxide particles in Chapter 18 and chemical exchange shift transfer in Chapter 19 for identification and monitoring of brain tumors after treatment). The references are mostly up to date.

Brain Tumor Imaging includes good, comprehensive coverage of past, current, and future MRI technologies for primary brain tumors, particularly gliomas. However, in oncologic patients with brain tumors, most will have brain metastases as well. Although this book indeed mentions cerebral metastases from breast cancer (chapter 6) and from testicular cancer (chapter 8 ), as well as covering paraneoplastic effects (chapter 14), the coverage of metastasis is no more than a broad overview. In addition, although important topics such as posttreatment effects, MRI perfusion, diffusion, arterial spin-labeling, tractography, spectroscopy, high field strength, genomics, and new contrast media (ultrasmall superparamagnetic iron oxide particles) are covered in more than a single chapter each, coverage of the classic PET and SPECT molecular imaging with capabilities at subnanomolar levels is restricted mostly to one chapter, which describes several cerebral metastases.

This book is adequate for neuroradiology fellows in training who will practice mostly MRI with advanced options. Readers who desire a more thorough understanding of the modern molecular imaging of brain tumors should additionally consult other molecular imaging treatises and the vast literature on recent advances in scintigraphic studies.

\author{
Franklin Wong \\ M.D. Anderson Cancer Center \\ 1400 Pressler St. \\ Houston, TX 77030 \\ E-mail: fwong@mdanderson.org
}

